EPJ Web of Conferences 86,00062 (2015)

DOI: $10.1051 /$ epjconf/ 20158600062

(C) Owned by the authors, published by EDP Sciences, 2015

\title{
Fusion and quasi-fission in heavy systems with the microscopic time- dependent energy density functional theory
}

\author{
Kouhei Washiyama ${ }^{1, a}$ \\ ${ }^{1}$ RIKEN Nishina Center, Wako 351-0198, Japan
}

\begin{abstract}
Fusion hindrance, where fusion probability in heavy systems is strongly hindered compared with that in light and medium-mass systems, is analyzed by the microscopic time-dependent energy density functional theory. From trajectories obtained for fusion reactions, we extract nucleus-nucleus potential and one-body energy dissipation for the entrance channel of fusion reactions in heavy systems. We find that a barrier structure disappears and an increase behavior is observed in the obtained potential, which are different from the cases of light and medium-mass systems and of the frozen density approximation. We show that main contribution to extra-push energy comes from the increase of potential energy because of dynamical effects.
\end{abstract}

\section{Introduction}

The interplay between nuclear structure and dynamical effects is crucial to properly describing heavy-ion fusion reactions at energies around the Coulomb barrier. Coupledchannels calculations [1-4] have been widely used to quantitatively describe the entrance channel of fusion reactions in light and medium-mass systems, whose charge product $\left(Z_{1} Z_{2}\right)$ is less than 1600 . On the other hand, it has been observed in heavy systems (typically $Z_{1} Z_{2}>1600$ ) that the fusion probability is strongly hindered around the Coulomb barrier compared with that in $Z_{1} Z_{2}<1600$ systems [5]. This phenomenon is called fusion hindrance and an extra energy to be needed to make such systems to fuse is called extra-push energy [6]. Quasi-fission process, where a colliding system after a touching configuration reseparates to projectile-like and target-like fragments before forming a compound nucleus, is considered to be mostly responsible for this hindrance. For a better description of the reaction mechanism in heavy systems, a dynamical diffusion model using a macroscopic Langevin equation has been developed $[7,8]$.

Time-dependent energy density functional model, in nuclear physics often called time-dependent Hartree-Fock (TDHF) model [9-11], has been developed and widely used to describe a variety of low energy nuclear reactions [12-15]. It is well known that TDHF based on energy density functionals provides a unique tool for describing nuclear structure and nuclear reactions, that is, static and dynamical properties of atomic nuclei over the whole nuclear chart in a unified self-consistent framework. Along this line, extra-push energies and quasi-fission process have been analyzed in Refs. [16-18]. The density-constrained TDHF model [19], which combines TDHF dynamics with

\footnotetext{
a e-mail: kouhei.washiyama@riken.jp
}

energy minimization under constraints on the density obtained with TDHF, has been applied to obtain nucleusnucleus potentials for heavy systems [20, 21].

Recently, we have proposed a method to directly extract nucleus-nucleus potential and one-body energy dissipation from the relative motion of colliding nuclei to nuclear intrinsic excitations in fusion reactions from TDHF evolutions $[22,23]$. This method relies on the hypothesis that complex microscopic mean-field evolution of headon collisions can be accurately reduced to a simple onedimensional macroscopic evolution which obeys a classical Newton equation including potential and dissipation terms. In the present work, we apply this method to study the properties of potential and energy dissipation in the entrance channel of fusion reactions for heavy systems and to understand origins of fusion hindrance.

\section{Method}

In this section, we illustrate our method to extract nucleusnucleus potential and energy dissipation for fusion reactions from TDHF trajectories. Our method consists of four steps:

1. We solve TDHF equations for central collisions to obtain the total density $\rho(t)$ at each time until two nuclei substantially overlap.

2. We compute the relative distance $R(t)$ and associated momentum $P(t)$ from the total density with a suitable definition of the separation plane at which the total system is divided into two subsystems.

3. We assume that obtained collective coordinates $R$ and $P$ obey a classical equation of motion with a 


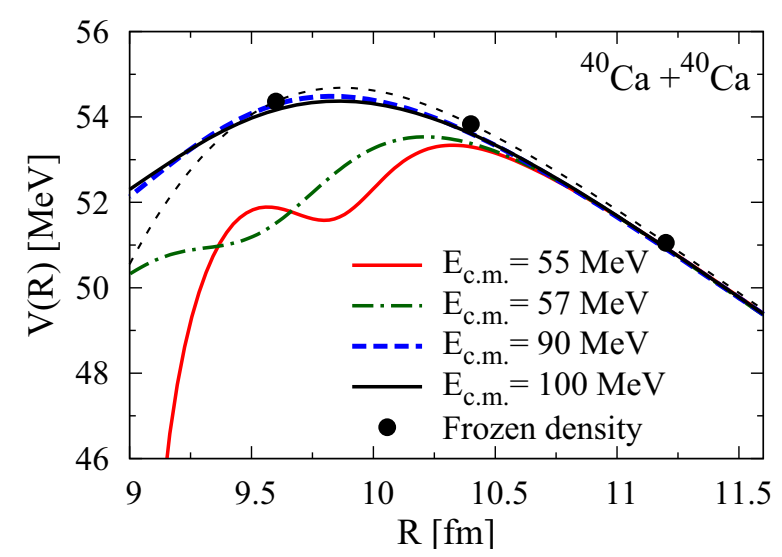

Figure 1. Nucleus-nucleus potential as a function of relative distance for the ${ }^{40} \mathrm{Ca}+{ }^{40} \mathrm{Ca}$ system extracted at different $E_{\mathrm{cm}}$. Filled circles denote the frozen density potential.

friction term,

$$
\begin{aligned}
& \frac{d R}{d t}=\frac{P}{\mu}, \\
& \frac{d P}{d t}=-\frac{d V}{d R}-\frac{d}{d R}\left(\frac{P^{2}}{2 \mu}\right)-\gamma \frac{d R}{d t},
\end{aligned}
$$

where $\mu, V$ and $\gamma$ denote the reduced mass, nucleusnucleus potential and friction coefficient, respectively. The friction term describes dissipation from the relative motion to intrinsic degrees of freedom.

4. In this equation, there are two unknown quantities $V$ and $\gamma$. We prepare a set of two equations from two trajectories of central collisions with two slightly different energies and by inverting this set of equations we obtain $\gamma$ and $d V / d R$.

We would like to note here that our extracted potential is based on neither sudden nor adiabatic approximation. Our extracted potential and friction automatically contain dynamical effects such as dynamical density evolution during collision.

To compute TDHF evolutions, we used the threedimensional TDHF code developed by P. Bonche and coworkers with SLy4d Skyrme parameter set [12] with the mesh size in space and time being $0.8 \mathrm{fm}$ and $0.45 \mathrm{fm} / c$, respectively. Details of the computations can be found in Refs. [22, 23]. In these references, we have investigated systematically fusion reactions in light and medium-mass systems. Here, we show one example in Fig. 1, where nucleus-nucleus potentials extracted at different centerof-mass energies $E_{\mathrm{cm}}$ are plotted as a function of $R$ for the ${ }^{40} \mathrm{Ca}+{ }^{40} \mathrm{Ca}$ system. As a reference, we plot by the filled circles the frozen density potential [24] that is calculated from the same energy density functionals as in TDHF with the densities of projectile and target nuclei frozen to their ground-state one, meaning that no dynamical effect is included during collision. We recall the main conclusions from Fig. 1: (i) At higher energies $\left(E_{\mathrm{cm}}=90\right.$ and $100 \mathrm{MeV}$ ), extracted potentials identify with the frozen density potential. This indicates that the system does not

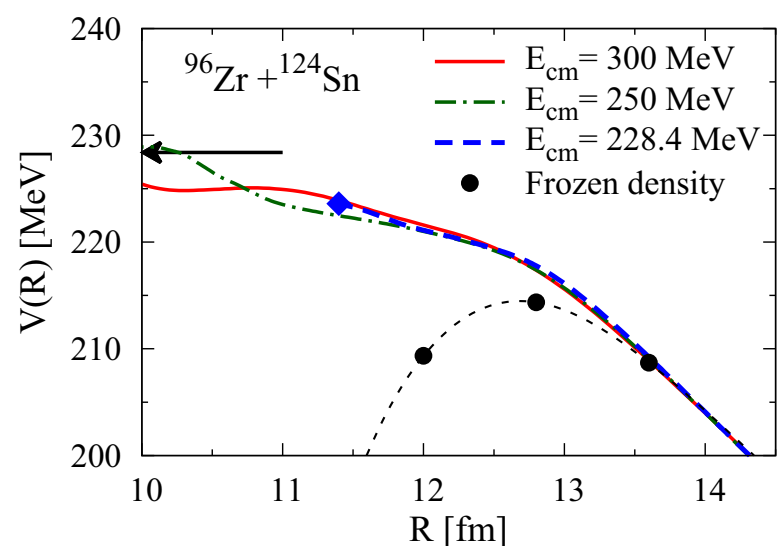

Figure 2. Same as Fig. 1, but for the ${ }^{96} \mathrm{Zr}+{ }^{124} \mathrm{Sn}$ system. The black arrow indicates the fusion threshold energy of this system, 228.4 MeV. The blue filled diamond indicates the relative distance where extraction of the potential stops (see text for detail).

have enough time to evolve and that a sudden approximation is valid. (ii) At energies near the Coulomb barrier $\left(E_{\mathrm{cm}}=55\right.$ and $\left.57 \mathrm{MeV}\right)$, extracted potentials show strong energy dependence and induce a reduction of the barrier height from the potential barrier extracted at higher energies and from the frozen density potential. This dynamical reduction effect leads to a good agreement with the barrier height obtained from experimental data.

In the next section, we apply this method and show the results for the ${ }^{96} \mathrm{Zr}+{ }^{124,132} \mathrm{Sn}$ and ${ }^{96} \mathrm{Zr}+{ }^{136} \mathrm{Xe}$ systems.

\section{Result}

Figure 2 shows extracted nucleus-nucleus potentials as a function of $R$ for the entrance channel of fusion reactions for the ${ }^{96} \mathrm{Zr}+{ }^{124} \mathrm{Sn}$ system $\left(Z_{1} Z_{2}=2000\right)$ at three $E_{\mathrm{cm}}$ and with the frozen density approximation. Here, we define fusion in TDHF as a reaction where the colliding system remains a compact shape for a long time, say $1200 \mathrm{fm} / c$. Then, we define fusion threshold energy as the minimum energy for fusion. In this system, we found that the fusion threshold energy is $E_{\text {thres }}=228.4 \mathrm{MeV}$, which is indicated by the black arrow. Note that, for the case with $E_{\mathrm{cm}}=228.4 \mathrm{MeV}$, we stop extracting the potential at $R \sim 11.4 \mathrm{fm}$, which is indicated by the blue filled diamond in Fig. 2. The reason is that the relative velocity $\dot{R}$ becomes almost 0 at $R \sim 11.4 \mathrm{fm}$ and our extraction method through Eq. (1) does not properly work.

By comparing the obtained potentials in Fig. 2 with those in $Z_{1} Z_{2}<1600$ systems displayed in Fig. 1, we find two significant differences:

- For the ${ }^{96} \mathrm{Zr}+{ }^{124} \mathrm{Sn}$ system, while a barrier is observed in the frozen density potential at $R \sim 12.8 \mathrm{fm}$, no potential barrier is observed in the potentials extracted with TDHF at any $E_{\mathrm{cm}}$ and the potentials monotonically increase as $R$ decreases. For the ${ }^{40} \mathrm{Ca}+{ }^{40} \mathrm{Ca}$ system, a barrier is observed in any cases.

- Energy dependence of potential is observed in the ${ }^{40} \mathrm{Ca}+{ }^{40} \mathrm{Ca}$ system and has also been discussed in 


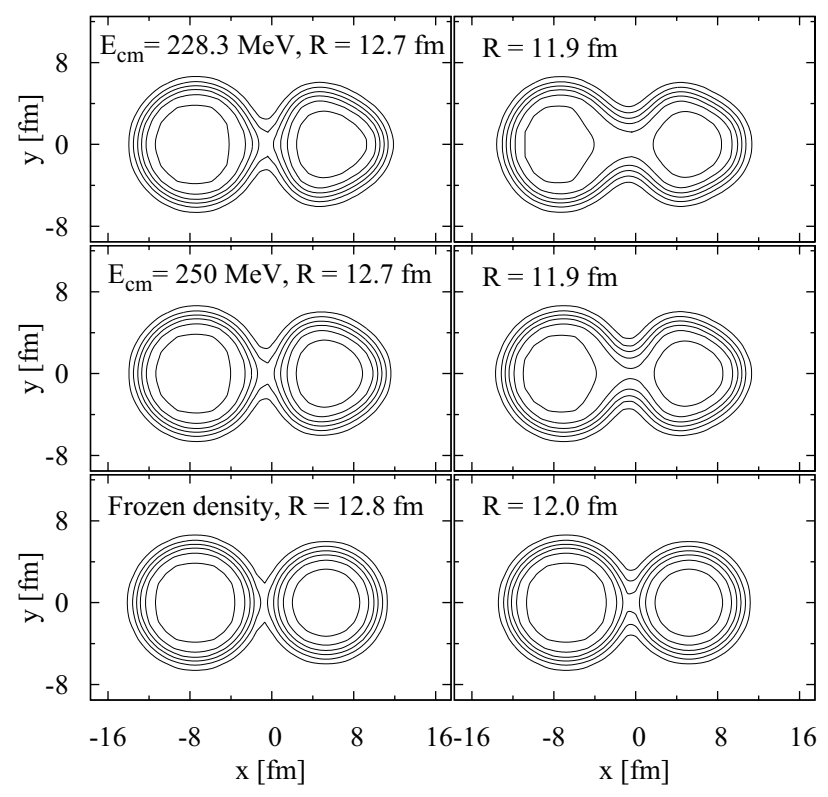

Figure 3. Density profiles $\rho(x, y, z=0)$ for the ${ }^{96} \mathrm{Zr}+{ }^{124} \mathrm{Sn}$ system at $E_{\mathrm{cm}}=228.3 \mathrm{MeV}$ (top) and $250 \mathrm{MeV}$ (middle) obtained with TDHF, and obtained from the frozen density approximation (bottom) for different relative distances indicated in each panel. Contour lines are plotted at each $0.025 \mathrm{fm}^{-3}$.

Refs. [25, 26], while it is less pronounced in the ${ }^{96} \mathrm{Zr}+{ }^{124} \mathrm{Sn}$ system around the frozen density potential barrier at $R \sim 12.8 \mathrm{fm}$.

We extracted potentials for other heavy systems and observed similar results [27]. Therefore, those pointed above can be typical features for heavy systems.

Figure 3 shows the density profiles at the entrance channel of central collisions for the ${ }^{96} \mathrm{Zr}+{ }^{124} \mathrm{Sn}$ system. Two energies $E_{\mathrm{cm}}=228.3 \mathrm{MeV}$ (top panels), just below the fusion threshold energy, and $250 \mathrm{MeV}$ (middle panels), and as a reference the frozen density approximation (bottom panels) are employed. Two relative distances $R \sim 12.8 \mathrm{fm}$ (left panels) and $12.0 \mathrm{fm}$ (right panels), which are around the frozen density barrier, are chosen. For the two energies, similar density profiles can be seen and are different from the frozen density case. Even in the case of $E_{\mathrm{cm}}=250 \mathrm{MeV}$, which is well above the fusion threshold energy, dynamical deformation and neck formation of the density are observed, leading to dynamical change in potential. This was not observed in the results of the ${ }^{40} \mathrm{Ca}+{ }^{40} \mathrm{Ca}$ system at energies well above the Coulomb barrier energy [22]. Analysis along this line is underway.

Next we show energy dissipation in heavy systems. TDHF includes one-body dissipation mechanism from the microscopic point of view because of the treatment of the self-consistent mean field. Dissipated energy from the relative motion to internal excitations is considered as one of the origins of extra-push energy. In our method, this energy can be calculated through the friction parameter by

$$
E_{\mathrm{diss}}[R(t)]=\int_{0}^{t} d t^{\prime} \gamma\left[R\left(t^{\prime}\right)\right]\left(\frac{d R}{d t}\right)^{2}
$$

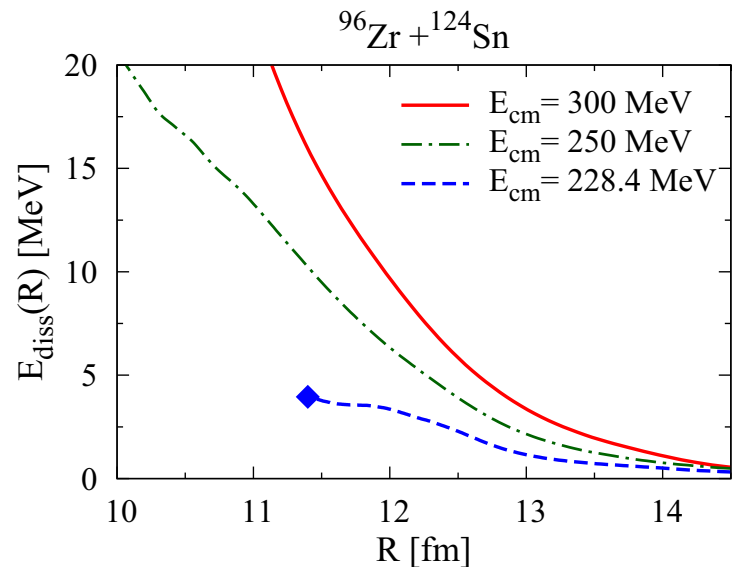

Figure 4. Dissipated energy calculated with Eq. (2) for the ${ }^{96} \mathrm{Zr}+{ }^{124} \mathrm{Sn}$ system.

Table 1. Fusion threshold energy $E_{\text {thres }}$, frozen density potential barrier $V_{\mathrm{FD}}$, extra-push energy $E_{\mathrm{extra}}$, increase of potential $\Delta V$, and dissipated energy $E_{\text {diss }}$ for the ${ }^{96} \mathrm{Zr}+{ }^{124,132} \mathrm{Sn}\left(Z_{1} Z_{2}=2000\right)$ and ${ }^{96} \mathrm{Zr}+{ }^{136} \mathrm{Xe}\left(Z_{1} Z_{2}=2160\right)$ systems. All energies are in units of $\mathrm{MeV}$.

\begin{tabular}{cccccc}
\hline System & $E_{\text {thres }}$ & $V_{\text {FD }}$ & $E_{\text {extra }}$ & $\Delta V$ & $E_{\text {diss }}$ \\
\hline${ }^{96} \mathrm{Zr}+{ }^{124} \mathrm{Sn}$ & 228.4 & 214.4 & 14.0 & 9.2 & 4.0 \\
${ }^{96} \mathrm{Zr}+{ }^{132} \mathrm{Sn}$ & 226.2 & 213.6 & 12.6 & 7.2 & 4.7 \\
${ }^{96} \mathrm{Zr}+{ }^{136} \mathrm{Xe}$ & 246.8 & 231.0 & 15.8 & 9.3 & 5.4 \\
\hline
\end{tabular}

Figure 4 shows the dissipated energies calculated from Eq. (2) for the ${ }^{96} \mathrm{Zr}+{ }^{124} \mathrm{Sn}$ system with different $E_{\mathrm{cm}}$. In all the cases, the dissipated energy monotonically increases as colliding nuclei approach to each other.

Furthermore, we analyze the origin of the fusion hindrance. Following Refs. [16, 17], we define the extra-push energy $E_{\text {extra }}$ from TDHF calculations as the difference between the fusion threshold energy $E_{\text {thres }}$ and the frozen density potential barrier $V_{\mathrm{FD}}$, that is, $E_{\text {extra }}=E_{\mathrm{thres}}-V_{\mathrm{FD}}$. For this purpose, we used the TDHF trajectory at the fusion threshold energy. In this case, at the relative distance where we stop extracting potential and energy dissipation (hereafter we denote this relative distance as $R_{\text {stop }}$ ), because of small remaining kinetic energy, we can identify the origin of the extra-push energy obtained with TDHF as the dissipated energy $E_{\text {diss }}$ and change in potential energy $\Delta V$ at $R_{\text {stop }}$ from the frozen density potential barrier, that is, $\Delta V=V\left(R_{\text {stop }}\right)-V_{\mathrm{FD}}$. In Table 1 , we summarize the different energies obtained from the present analysis for the ${ }^{96} \mathrm{Zr}+{ }^{124,132} \mathrm{Sn}$ and ${ }^{96} \mathrm{Zr}+{ }^{136} \mathrm{Xe}$ systems. In any systems, these energies were obtained from the TDHF trajectory at $E_{\text {thres. }}$. As mentioned above, increase of potential and dissipated energies are calculated at $R_{\text {stop }}$. Finite values $(\sim 15 \mathrm{MeV})$ of the extra-push energies are obtained in any systems. We confirm that the fusion hindrance exists in these systems, which is consistent with earlier experimental observations [5]. It is clearly seen in Table 1 that the increase of potential $\Delta V$ is about two times larger than the dissipated energy for all the systems. We conclude from this analysis that the main contribution to the extra- 
push energy is the increase of extracted potential. Energy dissipation plays an important role as expected, but is not sufficient to explain the fusion hindrance of those systems.

\section{Summary}

We have studied the fusion hindrance in heavy systems with a microscopic TDHF model. We have applied our method of directly extracting nucleus-nucleus potential and energy dissipation to fusion reactions in heavy systems. Our method is based on the assumption that complex mean-field dynamics can be reduced to classical equation of motion with suitable choice of collective coordinate. We show that no potential barrier is observed and the obtained potential monotonically increases in the ${ }^{96} \mathrm{Zr}+{ }^{124} \mathrm{Sn}$ system. These behaviors are different from that in light and medium-mass systems and from the frozen density potential. Furthermore, we have analyzed the ${ }^{96} \mathrm{Zr}+{ }^{132} \mathrm{Sn}$ and ${ }^{96} \mathrm{Zr}+{ }^{136} \mathrm{Xe}$ systems. We have calculated the extra-push energies of those systems and concluded that main contribution to the extra-push energy is from the increase of potential energy inside the frozen potential barrier.

\section{Acknowledgements}

The author is supported by the Special Postdoctoral Researcher Program of RIKEN.

\section{References}

[1] A. B. Balantekin and N. Takigawa, Rev. Mod. Phys. 70, 77 (1998).

[2] M. Dasgupta, D. J. Hinde, N. Rowley, and A. M. Stefanini, Annu. Rev. Nucl. Part. Sci. 48, 401 (1998).

[3] K. Hagino and N. Takigawa, Prog. Theor. Phys. 128, 1061 (2012).

[4] B. B. Back, H. Esbensen, C. L. Jiang, and K. E. Rehm, Rev. Mod. Phys. 86, 317 (2014).

[5] C.-C. Sahm et al., Nucl. Phys. A 441, 316 (1985).

[6] W. J. Swiatecki, Phys. Scripta 24, 113 (1981); Nucl. Phys. A 376, 275 (1982).
[7] V. Zagrebaev and W. Greiner, J. Phys. G 31, 825 (2005).

[8] Y. Aritomo, K. Hagino, K. Nishio, and S. Chiba, Phys. Rev. C 85, 044614 (2012).

[9] P. Bonche, S. E. Koonin, and J. W. Negele, Phys. Rev. C 13, 1226 (1976).

[10] H. Flocard, S. E. Koonin, and M. S. Weiss, Phys. Rev. C 17, 1682 (1978).

[11] J. W. Negele, Rev. Mod. Phys. 54, 913 (1982).

[12] K.-H. Kim, T. Otsuka, and P. Bonche, J. Phys. G 23, 1267 (1997).

[13] T. Nakatsukasa and K. Yabana, Phys. Rev. C 71, 024301 (2005).

[14] J. A. Maruhn, P.-G. Reinhard, P. D. Stevenson, and M. R. Strayer, Phys. Rev. C 74, 027601 (2006).

[15] A. S. Umar and V. E. Oberacker, Phys. Rev. C 73, 054607 (2006).

[16] C. Simenel, Eur. Phys. J. A 48, 152 (2012).

[17] L. Guo and T. Nakatsukasa, Eur. Phys. J. Web of Conference 38, 09003 (2012).

[18] A. Wakhle, C. Simenel, D. J. Hinde, M. Dasgupta, M. Evers, D. H. Luong, R. du Rietz, and E. Williams, Phys. Rev. Lett. 113, 182502 (2014).

[19] A. S. Umar and V. E. Oberacker, Phys. Rev. C 74, 021601(R) (2006)

[20] A. S. Umar, V. E. Oberacker, J. A. Maruhn, and P.-G. Reinhard, Phys. Rev. C 81, 064607 (2010).

[21] V. E. Oberacker, A. S. Umar, J. A. Maruhn, and P.-G. Reinhard, Phys. Rev. C 82, 034603 (2010).

[22] K. Washiyama and D. Lacroix, Phys. Rev. C 78, 024610 (2008).

[23] K. Washiyama, D. Lacroix and S. Ayik, Phys. Rev. C 79, 024609 (2009).

[24] C. Simenel, M. Dasgupta, D. J. Hinde, and E. Williams, Phys. Rev. C 88, 064604 (2013).

[25] L. C. Chamon et al., Phys. Rev. C 66, 014610 (2002).

[26] A. S. Umar, C. Simenel, and V. E. Oberacker, Phys. Rev. C 89, 034611 (2014).

[27] K. Washiyama, to be published. 